\title{
Return to the emergency department after ventricular shunt evaluation
}

\author{
Samir Sarda, BS, ${ }^{1}$ Harold K. Simon, MD, MBA, ${ }^{3}$ Daniel A. Hirsh, MD, ${ }^{4}$ Andrew Wang, BS, ${ }^{1}$ \\ R. Shane Tubbs, MS, PA-C, PhD, ${ }^{5}$ and Joshua J. Chern, MD, PhD ${ }^{1,2}$ \\ ${ }^{1}$ Pediatric Neurosurgery Associates and 4Pediatric Emergency Medicine Associates, Children's Healthcare of Atlanta; \\ Departments of ${ }^{2}$ Neurosurgery and ${ }^{3}$ Pediatrics, Emory University, Atlanta, Georgia; and ${ }^{5}$ Division of Neurosurgery, \\ Department of Surgery, University of Alabama at Birmingham, Alabama
}

OBJECTIVE Patients with CSF shunts are medically complex and frequently present to the emergency department (ED) with suspected shunt malfunction. After adequate evaluation in the ED and proper disposition, some patients return to the ED within a short period of time. In this study, the authors examined the reasons for ED revisits within 7 days of the index ED visit to discern possible preventable returns.

METHODS There were 3080 index ED visits made by patients with shunted hydrocephalus between 2010 and 2013. Index ED visits preceded by another ED visit or neurosurgical procedure within 60 days were excluded. Index ED visits for reasons unrelated to shunt function and those that led directly to admissions and shunt revision surgeries were also excluded. The remaining 1509 ED visits were eligible for analysis in this study. Final dispositions from the index ED visit included home (1176 cases), admission to the neurosurgery service for observation (134 cases), and admission to other services (199 cases). Subsequent events within 7 days, including ED revisits, hospital admissions, and shunt-related surgery were recorded, and reasons for the ED revisits were categorized based on whether the visit was related to shunt function concerns. Clinical and socioeconomic factors were analyzed for their association with ED revisits by using statistical methods.

RESULTS Of the 1176 patients discharged home from the ED after shunt function evaluation, $101(8.6 \%)$ returned to the ED within 7 days. Of the 134 patients admitted to the neurosurgery service for observation only, $8(6.0 \%)$ returned to the ED within 7 days of discharge. Of the 199 patients admitted to hospital services other than neurosurgery, $13(6.5 \%)$ returned to the ED within 7 days of discharge. The reasons for ED revisits vary (total of 122 visits combining the 3 groups), but at least $60 \%$ of the revisits were clearly unrelated to shunt function. A younger age, daytime arrival to the ED, and living within the metropolitan area were identified as risk factors for ED revisits.

CONCLUSIONS Children with CSF shunts are medically complex and use ED services often. After an index ED visit at which shunt function was deemed to be the chief concern, the purpose of the subsequent return to the ED within 7 days was often for complaints unrelated to shunt function. Caution is warranted when attempting to classify these complex patients as having potential preventable return-to-system events.

http://thejns.org/doi/abs/10.3171/2015.8.PEDS15309

KEY WORDS emergency department utilization; ED revisits; CSF shunt; return to system; hydrocephalus

$\mathrm{E}$ MERGENCY department (ED) revisits are often perceived as undesirable by patients, physicians, and administrators alike. These revisits together with hospital readmissions are 2 commonly used quality measures for health care delivery. Multiple studies have attempted to identify system- and patient-level factors that are related to increased ED revisits, often finding significant correlations with patient age, chronic conditions, and higher disease severity scores.118 Some disease entities, such as sickle cell disease ${ }^{1,8}$ and mental illness, ${ }^{1,11,14}$ were also found to be highly associated with ED utilization.

Children with CSF shunts are medically complex, have a chronic condition, and usually present with a high disease severity score., ${ }^{921,13}$ They often require multiple surgical interventions, and shunt surgery in particular has a documented high complication rate. ${ }^{14,16}$ Interestingly, to the best of our knowledge, no studies have directly identified CSF shunt patients as a group with an increased risk for

ABBREVIATIONS ED = emergency department.

SUBMITTED May 18, 2015. ACCEPTED August 24, 2015.

INCLUDE WHEN CITING Published online December 18, 2015; DOI: 10.3171/2015.8.PEDS15309. 
ED revisits. ${ }^{1,2,4}$ Since most of the studies performed thus far have been based on large administrative databases, one of the probable explanations for the absence of studies identifying these patients as a group with an increased risk for ED visits is the lack of specificity in diagnostic coding. The final diagnosis at revisits can be coded as "seizures," "headache," or "chronic disease," to name just a few examples we observed in the administrative database. Furthermore, despite the high acuity of these patients, they make up only a very small portion of the overall number of ED visits ${ }^{3}$ and thus can be overlooked.

Past studies related to CSF shunt patients in the ED setting have largely focused on recognizing signs and symptoms of shunt malfunction ${ }^{4,6,7,10,17}$ and the appropriateness of imaging utilization..$^{5,15}$ Herein, we conducted a singleinstitution study to document the clinical course of children after ED evaluation, with a focus on ED revisits. We first document the disposition of patients at the conclusion of the index ED visit, and then we describe 7-day ED revisits and readmissions. The reasons for each revisit were examined in detail in the hope of identifying potentially modifiable risk factors associated with preventable ED revisits in this particular group of patients.

\section{Methods}

\section{Patient Selection and Data Collection}

Children's Healthcare of Atlanta is a large pediatric health care system with 2 tertiary care pediatric hospitals, both of which are staffed with pediatric ED physicians and neurosurgeons. There were approximately 600,000 ED encounters within the 4-year study period between 2010 and 2013. We used 2 methods to identify patients with CSF shunts among the 600,000 ED visits. 1) The patient identity from each ED visit was cross-referenced with the operating room database to look for current procedural terminology (CPT) codes associated with previous shunt operations (62180-62258), if any. 2) A text search was performed for "shunt," "VPS," or "VAS" within the chief complaint data field originally documented by the ED triage nurse. The 2 methods generated significant overlap in the search results and enabled the detection of patients with a CSF shunt who had presented with a complaint unrelated to shunt function. A total of 3080 unique index ED visits were identified (Fig. 1).

We excluded 983 visits because the patient in these cases had an ED visit, hospital admission (including 23-hour observation), or shunt revision within 60 days prior to the index ED visit. In addition, ED visits for reasons clearly not related to shunt function were excluded $(n=154)$; these included presentations for feeding tube issues $(\mathrm{n}=38)$ or falls/traumatic injuries $(n=46)$. We excluded another 434 patients who had undergone shunt-related surgeries after being admitted to the hospital, since the occurrence of surgery is a significant event that would critically alter the subsequent clinical course of these patients. Therefore, the included index ED visits $(n=1509)$ were all presumed to be shunt-related; each was preceded by a clinically unremarkable period of at least 2 months; and if the patient was admitted, none underwent shunt-related surgeries.

Demographic, socioeconomic, and clinical factors were collected prospectively in the hospital electronic medical records and administrative databases for each index ED visit. An outcome-reporting tool developed within the institution was used to capture subsequent events such as ED revisits, readmissions, and surgeries within 7 days of the index ED visit. The 7-day interval was chosen because in the current emergency medicine literature, a return to the system within a period of 3-15 days was most commonly reported.

\section{Determining Reasons for Revisit and Statistical Analysis}

Two pediatric emergency board-certified physicians (D.A.H. and H.K.S.) and 1 pediatric neurosurgeon (J.J.C.) conducted extensive chart reviews for all patients who revisited the ED after discharge $(\mathrm{n}=122)$. Each reviewer independently adjudicated on whether a revisit was for shunt-related concerns and assigned each revisit to 1 of 2 categories: 1) definitely related to shunt concerns, and 2) definitely not related to shunt function. A third category of "indeterminate" was purposely not offered to force a categorical decision. Special attention was paid to the clinical scenarios whenever there was disagreement.

The rates of revisit for the 3 groups of patients were compared using a contingency table. Univariate and multivariate logistic regressions were modeled to identify clinical, hospital, and socioeconomic factors that might be associated with 2 outcomes of interest: whether the patient returned to the ED (yes/no) and whether the patient returned with shunt-related issues within 7 days of the index ED visit (yes/no). The multivariate model was constructed using risk factors identified in the univariate analysis. Statistical significance was defined as a $\mathrm{p}$ value $\leq 0.05$ and was determined using SPSS (version 20, IBM Corp.).

\section{Results}

\section{Patient Characteristics}

A total of 961 patients made 1509 index ED visits in the study period. The mean age at the time of the ED visit was $8.2 \pm 5.9$ years old $(136<1$ year of age, 436 between 1 and 5 years, 389 between 5 and 10 years), $92.4 \%$ of patients were English speaking, and 50.9\% self-reported as white. Patients with a private insurance plan made up $40.0 \%$ of the population. Of the 1509 index visits, 615 (40.8\%) occurred between 7 AM and 3 PM (Table 1).

\section{Patient Dispositions From ED and Subsequent Events Within 7 Days}

Patient dispositions at the time of the index ED visit are summarized in Fig. 1. The patients evaluated in the ED and discharged home numbered 1176 . The average length of ED stay was 3.29 hours (median 2.97 hours). Patients were uniformly instructed to schedule a neurosurgery outpatient appointment as part of their follow-up care. In the subsequent 7-day period for this cohort, there were 101 ED revisits $(8.6 \%)$ that resulted in 24 readmissions to the neurosurgical service (20 shunt-related surgeries) and 26 admissions to other services.

Five hundred fifty patients were admitted to the neurosurgery service from the ED, but 416 of those admissions were followed by shunt surgeries and were therefore excluded from statistical analysis. The remaining 134 pa- 


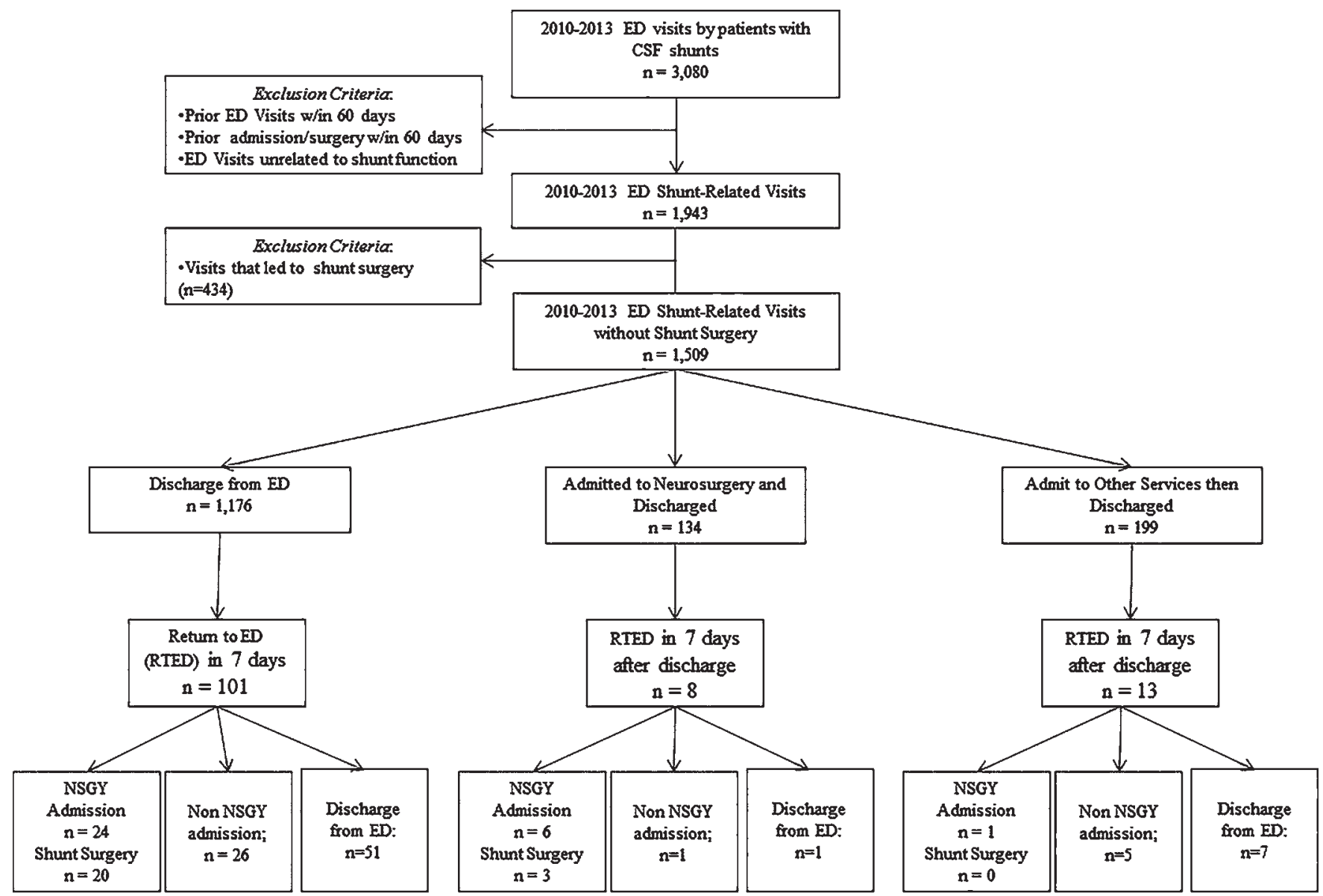

FIG. 1. Disposition and 7-day outcome of CSF shunt patients who were evaluated in the ED over a 4-year period. NSGY = neurosurgery; RTED = return to ED.

tients were discharged home when symptoms improved and did not undergo surgical intervention in the index hospitalization. The average length of the observation period was $2.13 \pm 3.23$ days (median 1.43 days). Of the patients who were observed and discharged without operation (n $=134)$, there were $8(6.0 \%)$ ED revisits within 7 days of discharge.

Lastly, 217 patients were admitted to nonneurosurgical services from the ED, with 18 (8.3\%) excluded for having undergone shunt-related surgery during their hospitalization. The most common admission services among this nonneurosurgical cohort were pediatrics $(n=103)$ and critical care $(\mathrm{n}=16)$. In the remaining patient population $(n=199)$, there were $13(6.5 \%)$ returns to the ED within 7 days of discharge.

\section{Reason for ED Revisit: Was It Related to Shunt Function?}

Reasons for the ED revisit were independently reviewed by 3 physicians, and they were categorized as 1) related to shunt function, 2) not related to shunt malfunction, or 3) equivocal. The 3 physicians completely agreed in 95 cases (78\%). Of these 95 cases, 21 were deemed related to shunt function, and 74 were not. Examples of the clinical scenarios are shown in Table 2.

The remaining 27 equivocal cases had 1 of the 3 physi- cians disagreeing, and interestingly the disagreeing physician was the neurosurgeon in only 3 cases. The most common clinical scenario that caused disagreements involved patients who had a history of chronic headache

TABLE 1. Patient characteristics and other variables associated with 1509 index ED visits

\begin{tabular}{lc}
\hline \multicolumn{1}{c}{ Variable } & Value \\
\hline Mean length of ED stay in mins (range) & $221 \pm 113(19.2-1132.8)$ \\
\hline Age in yrs (\%) & \\
$<1$ & $136(9.0)$ \\
$1-5$ & $436(28.9)$ \\
$5-10$ & $389(25.8)$ \\
\hline English as primary language (\%) & $1395(92.4)$ \\
\hline White (\%) & $768(50.9)$ \\
\hline Atlanta metropolitan zip code (\%) & $685(45.4)$ \\
\hline Medicaid/Medicare as primary payee (\%) & $905(60.0)$ \\
\hline Time of arrival: 7 AM-3 PM (\%) & $615(40.8)$ \\
\hline Admission to hospital (\%) & $333(22.1)$ \\
\hline ICU admission (\%) & $42(2.8)$ \\
\hline
\end{tabular}

ICU = intensive care unit.

* Mean value expressed with standard deviation. 
TABLE 2. Examples of clinical scenarios encountered at revisits and their adjudication

\begin{tabular}{|c|c|}
\hline Adjudication Result & Examples of Clinical Scenarios \\
\hline Revisits that were shunt related $(n=21)$ & $\begin{array}{l}\text { 1) Patient presented w/ headache at index ED visit but radiographic exam was negative; at revisit, radio- } \\
\text { graphic exam was consistent } w / \text { shunt malfunction. } \\
\text { 2) Patient complained of persistent headache at revisit \& was taken for shunt exploration. }\end{array}$ \\
\hline $\begin{array}{l}\text { Revisits that were not shunt related* } \\
\qquad(n=74)\end{array}$ & $\begin{array}{l}\text { 1) Patient presented w/ headache initially then re-presented w/ rash, vomiting, \& diarrhea. } \\
\text { 2) Patient revisited for feeding tube-related issues. } \\
\text { 3) Patient was asked to return when urine culture sent at index ED visit became positive. }\end{array}$ \\
\hline Equivocal cases $(n=27)$ & $\begin{array}{l}\text { 1) Patient w/ pseudotumor cerebri returned w/ complaints of persistent headache. } \\
\text { 2) Patient presented w/ increased seizure \& persistent seizure at index visit \& revisits. }\end{array}$ \\
\hline
\end{tabular}

* Agreed by all 3 reviewing physicians.

and who presented with persistent headache even though shunt malfunction had been ruled out at the index visit. The second most common scenario was the patient with known seizure disorders presenting with increased seizure frequency or severity.

Overall, $17 \%(21 / 122)$ of the revisits were deemed to be definitively related to shunt function, and $61 \%(74 / 122)$ of the revisits were definitively not related to shunt function. The proportion of revisits that may relate to shunt function concerns, including the equivocal cases was, at most, $39 \%$ (48/122).

\section{Statistical Analysis}

After the patient was discharged from the ED, neurosurgical service, or other inpatient service (Fig. 1), the rates of ED revisits within 7 days were $8.6 \%, 6.0 \%$ and $6.5 \%$, respectively, and the differences were not statistically significant ( $\mathrm{p}=0.349$, contingency table).

Univariate and multivariate analysis was used to look for associations among clinical and socioeconomic factors existing at the index ED visits and 2 categorical dependent variables: all ED revisits within 7 days $(n=122)$ and ED revisits with shunt function concerns $(n=48$; Tables 3 and 4). In the multivariate analysis, patients who had an increased risk for ED revisits were younger, non-English speaking, living within the Atlanta metropolitan area, and arriving during daytime hours (7 AM-3 PM). Besides patient age, all of the identified risk factors were socioeconomic and not clinical. When patients returned for shunt-related concerns (a subset within all ED revisits), the correlating risk factors identified were a young age and daytime arrival (OR 2.4 and 1.9, respectively).

\section{Discussion}

\section{Rationale for the Study}

In this study, we identified a large cohort of patients who had been evaluated in the ED for their shunt function over a 4-year period. The occurrence and reasons for ED revisits after the index visit were then studied. Our purpose was to identify potentially modifiable risk factors associated with preventable ED revisits in this particular group of patients. This study has 2 important features: First, because this is a single-institution study, we were able to obtain detailed longitudinal data to exclude all patients who had recent preceding clinical events. This is particularly important for CSF shunt patients as shunt surgery is known for its relatively high complication rate. Furthermore, a recent ED visit prior to the index ED visit is probably in itself a risk factor for an ED revisit. Second,

TABLE 3. Univariate analysis of clinical and socioeconomic factors associated with all returns and shunt-related returns to the ED within 7 days*

\begin{tabular}{|c|c|c|c|c|}
\hline \multirow[b]{2}{*}{ Independent Variable } & \multicolumn{2}{|c|}{$\begin{array}{l}\text { Dependent Variable: All Return Visits } \\
\qquad(n=122)\end{array}$} & \multicolumn{2}{|c|}{$\begin{array}{c}\text { Dependent Variable: Shunt-Related } \\
\text { Return Visits }(n=48)\end{array}$} \\
\hline & OR $(95 \% \mathrm{Cl})$ & p Value & OR $(95 \% \mathrm{Cl})$ & $p$ Value \\
\hline \multicolumn{5}{|l|}{ Age in yrs } \\
\hline$<1$ & $2.33(1.30-4.18)$ & 0.004 & $2.37(1.00-5.63)$ & 0.050 \\
\hline $1-5$ & $1.05(0.65-1.69)$ & 0.857 & $1.04(0.49-2.20)$ & 0.927 \\
\hline $5-10$ & $0.73(0.42-1.26)$ & 0.257 & $0.71(0.31-1.63)$ & 0.421 \\
\hline Length of ED stay in mins & $1.00(0.99-1.00)$ & 0.200 & $1.00(0.99-1.00)$ & 0.505 \\
\hline Non-English speaking & $2.03(1.13-3.63)$ & 0.017 & $0.77(0.22-2.65)$ & 0.678 \\
\hline White & $1.09(0.74-1.60)$ & 0.680 & $1.10(0.61-2.02)$ & 0.740 \\
\hline Atlanta metropolitan zip code & $1.71(1.16-2.52)$ & 0.006 & $1.33(0.74-2.40)$ & 0.346 \\
\hline Medicaid/Medicare as primary payee & $1.05(0.70-1.59)$ & 0.809 & $1.06(0.57-1.97)$ & 0.863 \\
\hline Time of arrival 7 AM-3 PM & $1.57(1.07-2.29)$ & 0.021 & $1.88(1.05-3.39)$ & 0.035 \\
\hline Admission to hospital & $0.61(0.33-1.13)$ & 0.117 & $1.43(0.38-5.35)$ & 0.593 \\
\hline ICU admission & $2.04(0.60-7.00)$ & 0.256 & $0.00(0.00-0.00)$ & 0.998 \\
\hline
\end{tabular}

* Bold values indicate statistical significance. 
TABLE 4. Multivariate analysis of clinical and socioeconomic factors associated with all returns and shunt-related returns to the ED within 7 days*

\begin{tabular}{|c|c|c|c|c|}
\hline \multirow[b]{2}{*}{ Independent Variable } & \multicolumn{2}{|c|}{$\begin{array}{l}\text { Dependent Variable: All Return Visits } \\
\qquad(n=122)\end{array}$} & \multicolumn{2}{|c|}{$\begin{array}{l}\text { Dependent Variable: Shunt-Related Return } \\
\text { Visits }(n=48)\end{array}$} \\
\hline & $\mathrm{OR}(95 \% \mathrm{Cl})$ & $p$ Value & $\mathrm{OR}(95 \% \mathrm{Cl})$ & p Value \\
\hline Age $<1 \mathrm{yr}$ & $2.39(1.44-3.96)$ & 0.001 & $2.41(1.14-5.11)$ & 0.021 \\
\hline Non-English speaking & $2.14(1.23-3.73)$ & 0.007 & & \\
\hline Atlanta metropolitan zip code & $1.69(1.15-2.48)$ & 0.007 & & \\
\hline Time of arrival $7 \mathrm{AM}-3 \mathrm{PM}$ & $1.56(1.07-2.27)$ & 0.021 & $1.90(1.07-3.40)$ & 0.030 \\
\hline
\end{tabular}

* Bold values indicate statistical significance.

in contrast to studies based on national databases or a single institutions that examine the ED revisits of all patients, we limited our study to a patient cohort with a particular clinical characteristic (having a CSF shunt) that is generally considered to be unique and high risk..$^{1,4,19}$ This study is similar in design and rationale to studies evaluating ED utilization by patients with sickle cell disease or psychiatric disorders; however, to our knowledge, a study based on CSF shunt patients has not been conducted.

\section{Results and Insights From This Study}

We first documented the rate of revisits within 7 days after an index ED visit by a CSF shunt patient as $8.1 \%$ $(122 / 1509)$, which is substantially higher than other rates of pediatric ED revisits for other indications. ${ }^{2,4}$ Not surprisingly, both socioeconomic and clinical factors may contribute to an increased risk of ED revisits. In particular, speaking a language other than English, proximity to the hospital, and a younger patient age were identified as significant risk factors in this and other studies. . $^{1,4,8,19}$ The finding of a correlation between increased revisits and the time of arrival was interesting and should be explored further. Notably, daytime arrival was identified as a risk factor in at least 1 other study based on general pediatric ED visits. ${ }^{4}$ Lastly, we found that proximity to the hospital seems to influence the likelihood of revisits, which is intuitive. Together, these findings suggest that despite the important clinical characteristic of having a CSF shunt, the phenomenon of ED revisits by patients with these shunts is strongly influenced by socioeconomic factors. ${ }^{1,8}$

Despite the heightened awareness of signs and symptoms of shunt malfunction, many patients presented with other unrelated complaints, reflecting the fact that these patients are complex and often have other medical comorbidities. Our results showed that at least $60 \%$ of patients, and maybe more, had a final diagnosis at the revisit that was clearly not related to shunt function. This result implies 2 opportunities for improvements. One is for the neurosurgical services: how can shunt function be better determined for the $40 \%$ of patients who revisited because of shunt malfunction? The other is for the ED physicians, who need to guard against excessive focus on shunt malfunction as the sole etiology to explain presenting symptoms. Consequently, if one were to design a protocol to decrease unplanned returns for CSF shunt patients, it would definitively require a multidisciplinary approach besides input from the neurosurgical team.

Our data also demonstrated the difficulty in adjudicat- ing whether a visit was related to shunt malfunction even with a detailed chart review. For example, in the case of a child who presented with an isolated, partial sixth nerve palsy in the absence of any other symptoms, 1 physician believed the symptoms could be attributed to shunt malfunction, whereas the other 2 physicians disagreed. In this study, we intentionally invited physicians who were not neurosurgeons but who had taken care of neurosurgical patients to serve as reviewers. We did so because most of the policy making regarding surgical outcomes at the hospital, state, and federal levels has been done by nonsurgeons. Our study indicates that ambiguous cases may account for $22 \%$ of all cases (27 of 122). Therefore, one should exercise caution when large database decisions are made to classify such complex patients as having "preventable returns" or even "related returns."

\section{Limitations of This Study}

This retrospective descriptive study has some important limitations. First, the retrospective nature of the study was inadequate to address the exact admission and discharge criteria at the index ED visit and also did not define what constitutes an adequate workup for children with putative CSF shunt malfunction. For example, while a majority of children underwent imaging studies while in the ED $(n=1417)$, the exact breakdowns between CT and shunt series were not known. This study also did not address the types of therapeutic interventions that the patients may have received in the ED. While the neurosurgeons were involved in every admission to the neurosurgical service, their participation in the ED evaluation process was not well documented. However, this study had the advantage of evaluating actual practice by a large cohort of pediatric ED physicians caring for these patients. Second, the neurosurgical expertise for these patients came from a 5 -physician group in a single practice. Third, this study is based on a single pediatric health care system, and the possibility exists that patients may have presented to other facilities for care. Of note, however, our hospital is the only pediatric-specific facility in the state with a pediatric intensive care unit, so it is unlikely that any significant ED encounters or admissions occurred for these complex patients at an unrelated facility.

\section{Conclusions}

Children with CSF shunts are medically complex and use ED services often. After an index ED visit at which 
shunt function is deemed to be the chief concern, a subsequent ED revisit within 7 days is often for complaints unrelated to shunt function. Caution is warranted when attempting to classify these complex patients as having potential preventable return-to-system events.

\section{References}

1. Akenroye AT, Thurm CW, Neuman MI, Alpern ER, Srivastava G, Spencer SP, et al: Prevalence and predictors of return visits to pediatric emergency departments. J Hosp Med 9:779-787, 2014

2. Alessandrini EA, Lavelle JM, Grenfell SM, Jacobstein CR, Shaw KN: Return visits to a pediatric emergency department. Pediatr Emerg Care 20:166-171, 2004

3. Chern JJ, Macias CG, Jea A, Curry DJ, Luerssen TG, Whitehead WE: Effectiveness of a clinical pathway for patients with cerebrospinal fluid shunt malfunction. J Neurosurg Pediatr 6:318-324, 2010

4. Cho CS, Shapiro DJ, Cabana MD, Maselli JH, Hersh AL: A national depiction of children with return visits to the emergency department within 72 hours, 2001-2007. Pediatr Emerg Care 28:606-610, 2012

5. Cohen JS, Jamal N, Dawes C, Chamberlain JM, Atabaki SM: Cranial computed tomography utilization for suspected ventriculoperitoneal shunt malfunction in a pediatric emergency department. J Emerg Med 46:449-455, 2014

6. Garton HJ, Kestle JR, Drake JM: Predicting shunt failure on the basis of clinical symptoms and signs in children. J Neurosurg 94:202-210, 2001

7. Iskandar BJ, Tubbs S, Mapstone TB, Grabb PA, Bartolucci AA, Oakes WJ: Death in shunted hydrocephalic children in the 1990s. Pediatr Neurosurg 28:173-176, 1998

8. Jacobstein CR, Alessandrini EA, Lavelle JM, Shaw KN: Unscheduled revisits to a pediatric emergency department: risk factors for children with fever or infection-related complaints. Pediatr Emerg Care 21:816-821, 2005

9. Kim TY, Stewart G, Voth M, Moynihan JA, Brown L: Signs and symptoms of cerebrospinal fluid shunt malfunction in the pediatric emergency department. Pediatr Emerg Care 22:28-34, 2006

10. McNatt SA, Kim A, Hohuan D, Krieger M, McComb JG: Pediatric shunt malfunction without ventricular dilatation. Pediatr Neurosurg 44:128-132, 2008

11. Newton AS, Rosychuk RJ, Dong K, Curran J, Slomp M, McGrath PJ: Emergency health care use and follow-up among sociodemographic groups of children who visit emergency departments for mental health crises. CMAJ 184:E665E674, 2012

12. Piatt JH Jr: Thirty-day outcomes of cerebrospinal fluid shunt surgery: data from the National Surgical Quality Improvement Program-Pediatrics. J Neurosurg Pediatr 14:179-183, 2014

13. Piatt JH Jr, Freibott CE: Quality measurement in the shunt treatment of hydrocephalus: analysis and risk adjustment of the Revision Quotient. J Neurosurg Pediatr 14:48-54, 2014

14. Roberts YH, Huang CY, Crusto CA, Kaufman JS: Health, emergency department use, and early identification of young children exposed to trauma. J Emerg Med 46:719-724, 2014

15. Sellin JN, Cherian J, Barry JM, Ryan SL, Luerssen TG, Jea A: Utility of computed tomography or magnetic resonance imaging evaluation of ventricular morphology in suspected cerebrospinal fluid shunt malfunction. J Neurosurg Pediatr 14:160-166, 2014

16. Simon TD, Riva-Cambrin J, Srivastava R, Bratton SL, Dean JM, Kestle JR: Hospital care for children with hydrocephalus in the United States: utilization, charges, comorbidities, and deaths. J Neurosurg Pediatr 1:131-137, 2008

17. Vinchon M, Fichten A, Delestret I, Dhellemmes P: Shunt revision for asymptomatic failure: surgical and clinical results. Neurosurgery 52:347-356, 2003

18. Walsh-Kelly CM, Kelly KJ, Drendel AL, Grabowski L, Kuhn EM: Emergency department revisits for pediatric acute asthma exacerbations: association of factors identified in an emergency department asthma tracking system. Pediatr Emerg Care 24:505-510, 2008

19. Wolfson JA, Schrager SM, Khanna R, Coates TD, Kipke MD: Sickle cell disease in California: sociodemographic predictors of emergency department utilization. Pediatr Blood Cancer 58:66-73, 2012

\section{Disclosures}

The authors report no conflict of interest concerning the materials or methods used in this study or the findings specified in this paper.

\section{Author Contributions}

Conception and design: Chern. Acquisition of data: Simon, Hirsh, Wang. Analysis and interpretation of data: Sarda. Drafting the article: Sarda. Critically revising the article: Simon, Hirsh, Tubbs.

\section{Correspondence}

Joshua J. Chern, 5455 Meridian Mark Rd. NE, Ste. 540, Atlanta, GA 30342. email: joshua.chern@choa.org. 